ARTICLE

\title{
Large-Eddy Simulation of Plume Dispersion within a Regular Array of Cubic Buildings
}

\author{
Hiromasa NAKAYAMA* and Haruyasu NAGAI \\ Japan Atomic Energy Agency, 2-4 Shirakata, Tokai-mura, Naka-gun, Ibaraki-ken, 319-1195, Japan
}

\begin{abstract}
There is a potential problem that the intentional release of radioactive materials by terror attacks and the accidental spillage from the transportation or storage of flammable and toxic gas occur within populated urban area. For the assessment of human health hazard or the safety analysis of the hazardous gas, not only mean but also fluctuating concentrations should be estimated. Therefore, we perform Large-Eddy Simulation (LES) of turbulent flow and plume dispersion within a regular array of cubic buildings as idealized urban canopy and investigate the characteristics of mean and fluctuating concentrations. The geometry of the building array is $25 \times 7$ with $\lambda_{\mathrm{f}}=0.25$. Here, $\lambda_{\mathrm{f}}$ is the building frontal area index and defined as the ratio of the total frontal area of buildings to the plan area of the study site. The plume source is located at the ground surface behind the building in the 7th row of the building array. We can capture the unsteady behaviors of turbulent flow and plume dispersion within the building array and show the spatial distribution of mean and fluctuating concentrations.
\end{abstract}

KEYWORDS: LES, turbulent flow, dispersion, fluctuating concentration, buildings array

\section{Introduction}

An accurate analysis of plume dispersion is important for emergency responses against accidental or intentional release of hazardous and flammable materials within populated urban areas. For the assessment of human health hazard or the safety analysis of the hazardous gas, not only mean but also fluctuating concentrations should be estimated. Therefore, the dispersion characteristics of a plume through obstacle arrays have been examined mainly by field and wind tunnel experiments. For example, Macdonald et al. ${ }^{1)}$ and Sato et $a .^{2)}$ investigated the effect of building arrays on averaged characteristics of plume dispersion by a field experiment. Bezpalcova and Ohba, ${ }^{3)}$ and Oikawa and Meng ${ }^{4)}$ conducted wind tunnel experiments of plume dispersion within building arrays and investigated the effects of the building arrangement and obstacle density on the characteristics of mean and root mean square (r.m.s.) concentrations.

In this study, we perform a numerical simulation of plume dispersion within a regular array of cubic buildings as idealized urban canopy by Large-Eddy Simulation (LES) that can give detailed information on a concentration field. The objective of this study is to validate our LES model in comparison with the experimental data of Bezpalcova and $\mathrm{Ohba}^{3)}$ and investigate the distribution patterns of mean and fluctuating concentrations, and the characteristics of peak concentration within the building array.

\section{Numerical Model}

The governing equations for LES of atmospheric flow are the filtered continuity equation, the Navier-Stokes equation

*Corresponding author, E-mail:nakayama.hiromasa@jaea.go.jp

(C) 2011 Atomic Energy Society of Japan, All Rights Reserved. as follows;

$$
\begin{aligned}
& \frac{\partial \bar{u}_{i}}{\partial x_{i}}=0 \\
& \frac{\partial \bar{u}_{i}}{\partial t}+\bar{u}_{j} \frac{\partial \bar{u}_{i}}{\partial x_{j}}=-\frac{1}{\rho} \frac{\partial \bar{p}}{\partial x_{i}}+\frac{\partial}{\partial x_{j}} v\left(\frac{\partial \bar{u}_{i}}{\partial x_{j}}+\frac{\partial \bar{u}_{j}}{\partial x_{i}}\right)-\frac{\partial}{\partial x_{j}} \tau_{i j}+f_{i} \\
& \tau_{i j}=\overline{u_{i} u_{j}}-\bar{u}_{i} \bar{u}_{j}, \\
& \tau_{i j}-\frac{1}{3} \delta_{i j} \tau_{k k}=-v_{S G S} \overline{S_{i j}} \quad v_{S G S}=\left(C_{s} f_{s} \bar{\Delta}\right)^{2}\left(2 \overline{S_{i j}} \overline{S_{i j}}\right)^{\frac{1}{2}} \\
& \overline{S_{i j}}=\left(\partial \overline{u_{i}} / \partial x_{j}+\partial \overline{u_{j}} / \partial x_{i}\right) / 2
\end{aligned}
$$

and

$$
\bar{\Delta}=\left(\overline{\Delta_{x}} \overline{\Delta_{y}} \overline{\Delta_{z}}\right)^{\frac{1}{3}}
$$

where $u_{i}, t, p, \rho, v, \tau_{i j}$ and $f_{i}$ are the wind velocity, time, pressure, density, kinetic viscosity, subgrid-scale Reynolds stress and external force term, respectively. The subscript $i$ stands for coordinates (1: streamwise, 2: spanwise, and 3: vertical direction). Upper bars, $(\square$ ) denote application of the spatial filter. $\sigma_{i j}, v_{S G S}, C_{s}$ and $f_{s}$ are the Kronecker delta, the eddy viscosity coefficient, the model constant of flow field and Van Driest damping function, ${ }^{5)}$ respectively. $\bar{\Delta}$ denotes grid-filter width. In this LES model, the external force term proposed by Goldstein et al. ${ }^{6)}$ is applied for its computational stability for turbulent flow around bluff body. This force term, $f_{i}$ is incorporated into the Navier-Stokes equations to consider the building effects and can be assumed as the following expression;

$$
f_{i}=\alpha \int_{0}^{t} u_{i}\left(t^{\prime}\right) d t^{\prime}+\beta u_{i}(t), \quad \alpha<0, \beta<0,
$$


where $\alpha$ and $\beta$ are negative constants. The standard Smagorinsky model ${ }^{7)}$ with Van Driest damping function ${ }^{3)}$ is used because of its simplicity and low computational cost. $C_{s}$ is set to $0.12{ }^{8)}$

LES of plume dispersion is also computed by using the standard Smagorinsky model. ${ }^{7}$ The spatially filtered scalar conservation equations are presented by

$$
\begin{aligned}
& \frac{\partial \bar{c}}{\partial t}+\bar{u}_{j} \frac{\partial \bar{c}}{\partial x_{j}}=-\frac{\partial}{\partial x_{j}} s_{j}, \\
& s_{j}=\overline{u_{j} c}-\bar{u}_{j} \bar{c},
\end{aligned}
$$

and

$$
s_{j}=-\frac{v_{S G S}}{S c_{S G S}} \frac{\partial \bar{c}}{\partial x_{j}}
$$

where $s_{j}$ is the subgrid-scale scalar flux, which is also parameterized by an eddy viscosity model. The model constant, $S c_{s g s}$, is the turbulent Schmidt number and is set to a constant value, $0.5 .^{9)}$

Each equation is discretized using a finite-difference method based on staggered grid system where velocity components are defined at the boundary of a cell, while pressure and concentration are defined at the center of a cell. The coupling algorithm of velocity and pressure fields is based on the MAC method with the Adams-Bashforth scheme for time integration. ${ }^{10)}$ The Poisson equation is solved by the SOR method. For the spatial discretization in the governing equation of flow and the tracer transport, a second-order accurate central difference is used. For only advection term in a concentration field, Cubic Interpolated Pseudo-particle ${ }^{11,12)}$ is imposed. Reynolds number based on the cubical building height is almost 5,000.

\section{Test Calculations}

\section{The Wind Tunnel Experiment for Evaluating the Model Performance}

The experiment was carried out by Bezpalcova and $\mathrm{Ohba}^{3)}$ in the Boundary Layer Wind Tunnel at Wind Engineering Center of Tokyo Polytechnic University, Japan. The experimental set-up consists of buildings with dimensions: $70 \mathrm{~mm}$ (width), $70 \mathrm{~mm}$ (length), and $70 \mathrm{~mm}$ (height). They are arranged in the regularly aligned array with $\lambda_{f}=0.25$. Here, $\lambda_{f}$ is roughness density defined as the building frontal area index and defined as the ratio of the total frontal area of buildings to the plan area of the study site. There are 18 rows of buildings, each consists of 9 buildings. The ground-level point source is located at the center just behind the building of the 8th row and the 5th column of the arrays as show in Fig. 1. Here, the rows are numbered in increasing order in the streamwise direction from the leading edge of the array and the columns are numbered in increasing order in the spanwise direction from the right-hand side of the array when looking in the flow direction.

The approaching flow is generated by spires set up at the entrance of the wind tunnel section and roughness blocks. The profile of mean wind velocity can be approximated by a power law exponent of 0.25 . Concentration is measured by a

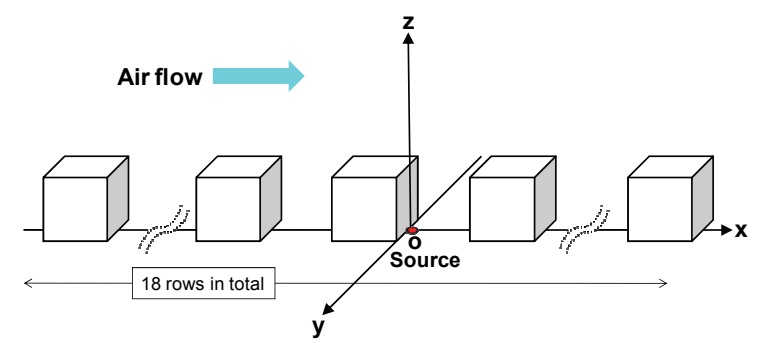

Fig. 1 Coordinate system and plume source location. These buildings depict the 5 th column of the arrays. The origin is the ground-level point source.

fast-response flame ionization detector. The vertical and spanwise profiles of mean and r.m.s. concentrations are shown by their experimental study.

In this study, to evaluate the model performance, we compare our LES results with these wind tunnel experimental data.

\section{Computational Settings}

In conducting LES of turbulent flow in the atmopshere, an approaching flow with turbulent fluctuations as the inlet of the model domain should be generated.

Figure 2 shows a schematic illustration of numerical model. Two computational domains are set up: The main region for a simulation of plume dispersion within a building array and the driver region for generating a spatially-developing turbulent boundary layer flow. First, a thick turbulent boundary layer flow is generated by incorporating the existing inflow turbulence generation method, i.e., Kataoka and Mizuno method ${ }^{13)}$ into an upstream small fraction of the driver region as shown in Fig. 2(a). Then, a strong wind velocity fluctuation is produced by a tripping fence and roughness blocks placed at the downstream of the recycle station as shown in Fig. 2(b). The fluctuating part of the velocity at the recycle station is recycled and added to the specified mean wind velocity at each time step by assuming that boundary layer thickness is constant within a driver section.

Calculations of both driver and main regions are conducted by the same model with different computational settings. As the boundary conditions, Sommerfeld radiation condition $^{14)}$ is imposed at the exit, a free-slip condition for streamwise and spanwise velocity components is imposed and vertical velocity component is 0 at the top, a periodic condition is imposed at the side, and a non-slip condition for each velocity component is imposed at the ground surface.

The size and the number of grid points for the driver region is $13.8 \delta \times 3.8 \delta \times 5.0 \delta$ ( $\delta$ : the height of a turbulent boundary layer thickness) and $460 \times 250 \times 100$ in streamwise, spanwise and vertical directions, respectively. The size and the number of grid points for the main region are $12.0 \delta \times 3.8 \delta \times 5.0 \delta$ and $1000 \times 250 \times 100$ in streamwise, spanwise and vertical directions, respectively.

In the main region, there are 25 rows of buildings and each consists of 8 buildings. The point source is located just behind the 8th row and the 4th column of the building arrays 
(a)

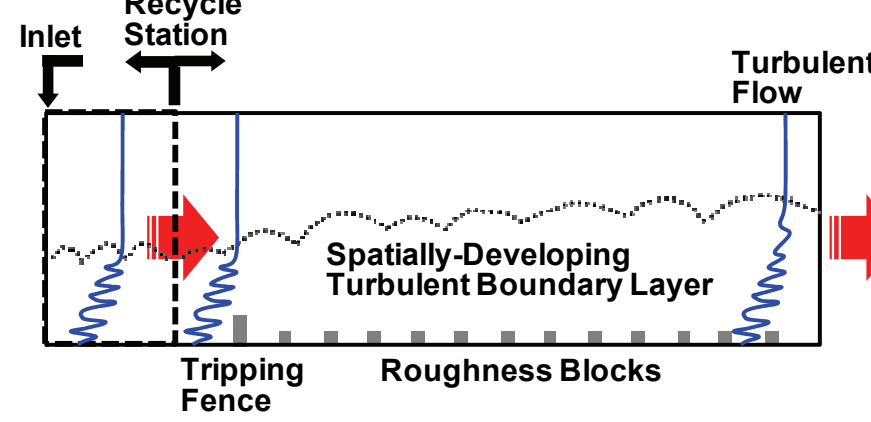

(b)

Turbulent Inflow

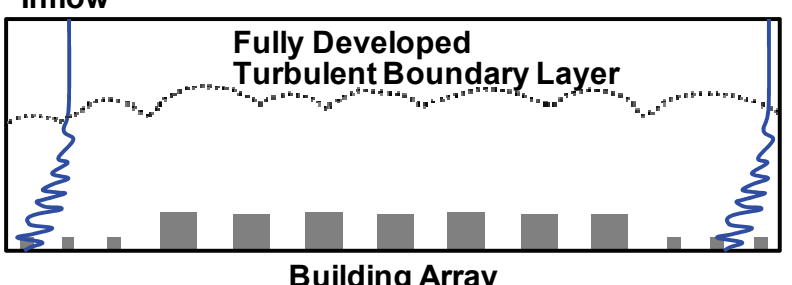

Fig. 2 Schematic of numerical model: (a) driver region for generating turbulent boundary layer flow and (b) main region for turbulent flow and plume dispersion within a building array

(a)

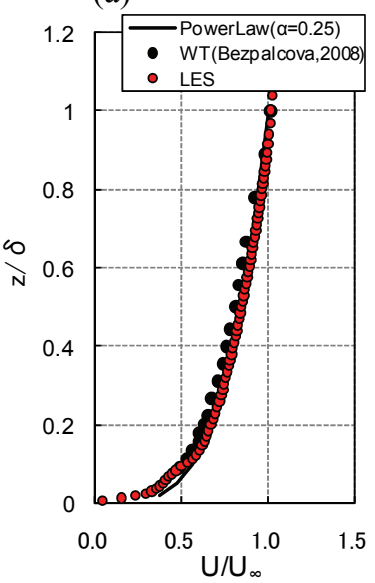

(b)

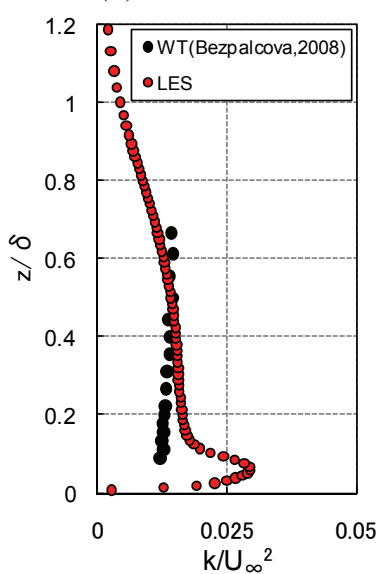

Fig. 3 Characteristics of approaching flow: (a) mean wind velocity and (b) turbulence kinetic energy

and at the ground surface. Each building of the array is resolved by $16 \times 16 \times 24$ grids in the streamwise, spanwise and vertical directions, respectively. At the inlet of the main region, the inflow turbulence data obtained near the exit of the driver region is imposed at each time step. In a concentration field, zero gradient is imposed at all the boundaries. As shown in Fig. 1, assuming that the location of a plume source point in the wind tunnel experiment is $\mathrm{x} / \mathrm{H}=0.0$, $\mathrm{y} / \mathrm{H}=0.0$ and $\mathrm{z} / \mathrm{H}=0.0(\mathrm{H}$ : a building height), that of a plume source point in this LES model is $\mathrm{x} / \mathrm{H}=0.0, \mathrm{y} / \mathrm{H}=-0.03$ and $\mathrm{z} / \mathrm{H}=0.0$. Because the number of grid points for individual cubic building is even number, the plume source position in the $\mathrm{y} / \mathrm{H}$ coordinate is slightly different from that in the experimental condition. Mean and r.m.s. concentrations of a plume are normalized by $\mathrm{H}$ and the source strength (Q).

\section{Results}

\section{Approaching Flow}

Figure 3 compares the LES results for mean wind velocity (U) and turbulence kinetic energy (k) with the experimental data of Bezpalcova and Ohba. ${ }^{3)}$ Mean wind velocity and turbulence kinetic energy are normalized by a free-stream velocity $\left(U_{\infty}\right)$, respectively. The height in the

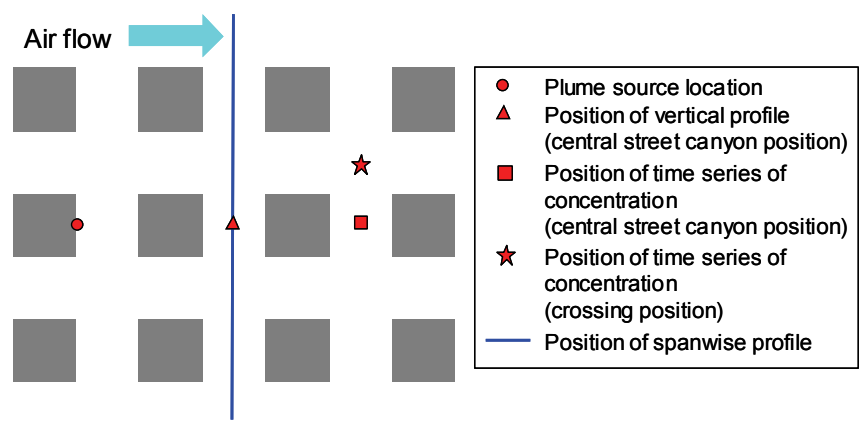

Fig. 4 Positions of the measurements of concentration for comparison of LES with the experiment. The circle depicts a source location. The triangle depicts the position of the vertical profile. The square and star symbols depict positions of time series of concentration. The blue line depicts the position of the spanwise profile.

plot is normalized with respect to $\delta$. $\delta$ corresponds to about $4.0 \mathrm{H}$.

It is found that mean wind velocity of LES is consistent with both wind velocity profile with a power law exponent of 0.25 and the experimental data. Turbulence kinetic energy by LES shows a peak near the ground surface by the influence of roughness blocks and then gradually decreases with height, while that of the experiment show a constant in the range of $0.1-0.7 \delta$ by the influence of spires set up at the wind tunnel entrance. Instead of this discrepancy turbulence kinetic energy by LES is similar in magnitude to that of the experiment. Because turbulent boundary layer flow is fully developed in this LES model, this slight discrepancy is negligible. Therefore, it is considered that approaching flow corresponding to the wind tunnel flow is successfully simulated in the driver region.

\section{Dispersion Characteristics}

Figure 4 shows positions of the measurements of concentration for comparison of LES with the experimental data.

Figure 5 shows the LES results for spanwise profiles of normalized mean $\left(\mathrm{C}_{\mathrm{ave}}\right)$ and r.m.s. $\left(\mathrm{C}_{\text {r.m.s. }}\right)$ concentrations. The location of the buildings is also shown. Mean concentration profile of the wind tunnel experiment shows a peak at the plume axis, thus, $\mathrm{y} / \mathrm{H}=0.0$. On the other hand, a peak of mean concentration profile of LES is located at $\mathrm{y} / \mathrm{H}=-0.5$. 
(a)

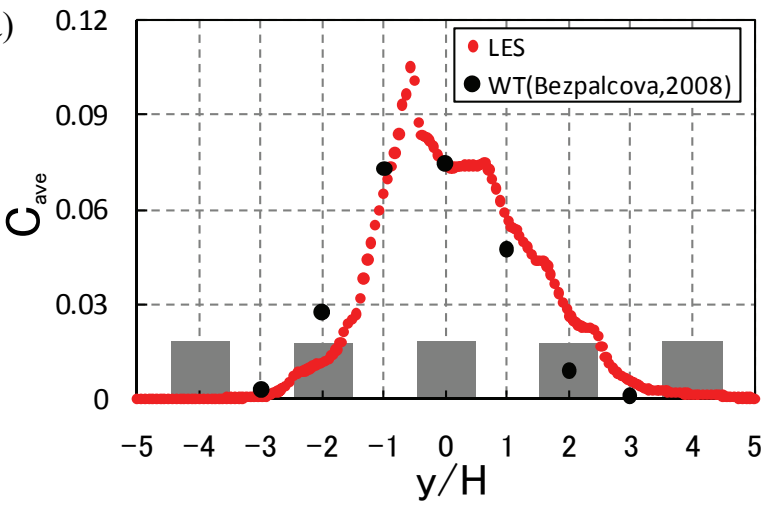

(b)

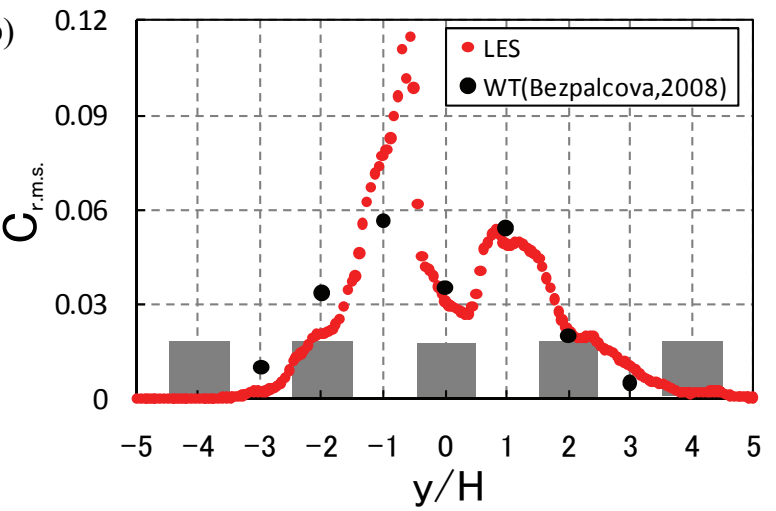

Fig. 5 Spanwise profiles of concentrations: (a) mean concentration and (b) r.m.s. concentration

However, mean concentration values of LES are generally similar in magnitude to those of the experiment. R.m.s. concentration profile of the experiment shows the local minimum at $\mathrm{y} / \mathrm{H}=0.0$ and the local maximum at $\mathrm{y} / \mathrm{H}=-1.0$ and 1.0. R.m.s. concentration profile of LES also shows such a tendency, but the value of the local maximum at $y / H=-1.0$ is overestimated. However, except the positions where LES data are overestimated, r.m.s. concentration values of LES are generally similar in magnitude to that of the experiment. Both mean and r.m.s. concentrations show an asymmetric pattern for LES data. The maximum value can be found at the left hand side $(\mathrm{y} / \mathrm{H}<0)$. This is due to even number of the computational cells and asymmetric placement of the source described in Section III. 2. However, the distributions rapidly become symmetric with downstream distance. The influence of these disagreements on the downward dispersion in the center axis is considered to be small.

Figure 6 shows the LES results for vertical profiles of normalized mean and r.m.s. concentrations by $\mathrm{H}$ and $\mathrm{Q}$ at the central street canyon position $(\mathrm{y} / \mathrm{H}=0)$ between 1 st and 2nd rows behind the source (see the triangle in Fig. 4). Mean concentration profile of LES shows almost a constant within building canopy (at heights less than $1.0 \mathrm{H}$ ) and gradually decrease above building canopy with height. Such a tendency is quantitatively consistent with the experiment. R.m.s. concentration profile of LES also shows a constant within building canopy, but increase just above building canopy and then decrease with height. Such a tendency is similar to the experiment.
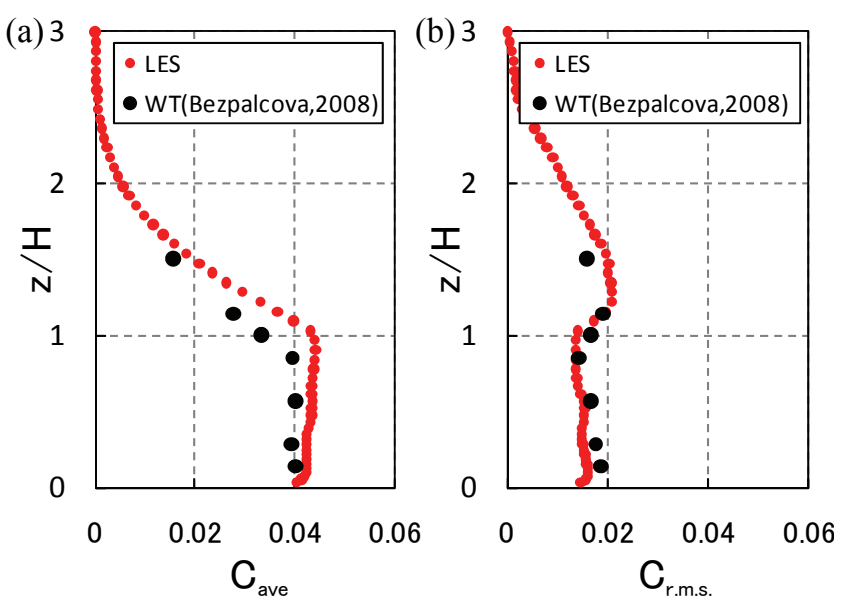

Fig. 6 Vertical profiles of concentrations: (a) mean concentration and (b) r.m.s. concentration

Although peak locations of the spanwise of mean and r.m.s. concentrations are a little different from those of the experiment and the peak value of r.m.s. concentration is overestimated, LES results are generally similar in magnitude to the experimental results. Furthermore, vertical profiles of mean and r.m.s. concentrations of LES are consistent with those of the experiment. Therefore, it is considered that the characteristics of mean and r.m.s. concentrations of a plume within a building array are successfully simulated by this LES model.

\section{Spatial Distributions of Concentrations}

Figure 7 shows the spatial distributions of mean and r.m.s. concentrations at the height of $0.1 \mathrm{H}$. It is found that mean concentration is distributed around the point source with wider spread by the influence of the buildings and then the spread is gradually getting narrower due to the sheltering effects of the building array. This distribution pattern of mean concentration is the same as Macdonald's field experiments. R.m.s. concentration is also distributed around the point source with wider spread, but its values is found to rapidly decrease with downwind distance.

Figure 8 shows the streamwise variation of mean and r.m.s. concentrations at the height of $0.1 \mathrm{H}$ at the plume axis $(y / H=0.0)$. It is found that mean concentration gradually decreases with downwind distance. R.m.s. concentration is found to rapidly decrease although its values increase within each central street canyon.

\section{Characteristics of Peak Concentration}

In case of release of toxic or flammable gases into the atmosphere, it is important to estimate instantaneous high concentrations. For this purpose, theoretical probability distributions of concentration fluctuation have been studied by many researchers. For example, Csanady ${ }^{15)}$ and Hanna ${ }^{16)}$ proposed theoretical models of the lognormal and exponential distributions for predicting concentration fluctuations of a plume, respectively, as follows. 
(a)

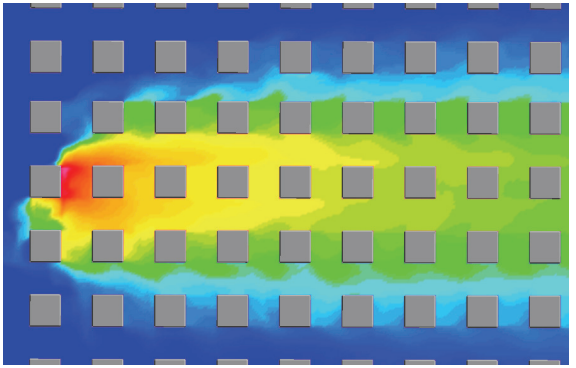

(b)

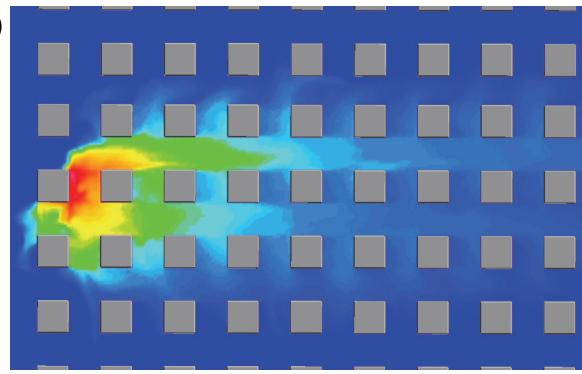

Fig. 7 Spatial distributions of concentrations at the height of $0.1 \mathrm{H}$ : (a) mean concentration and (b) r.m.s. concentration.

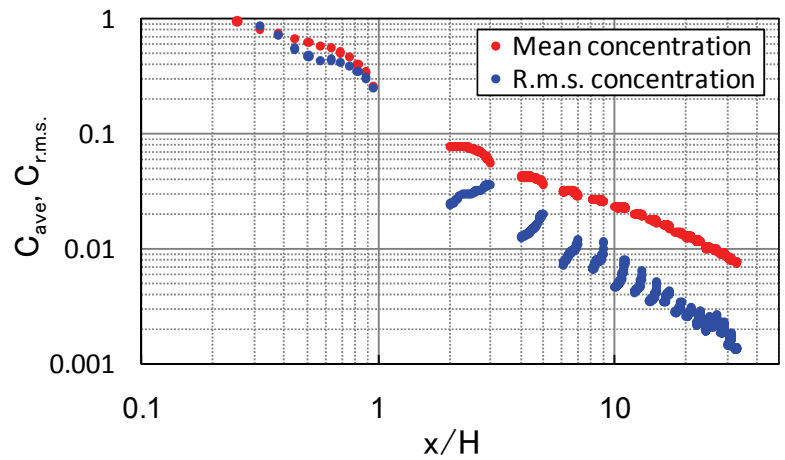

Fig. 8 Streamwise variations of mean and r.m.s. concentrations at the height of $0.1 \mathrm{H}$

Lognormal type: $P(c)=\frac{1}{2}\left[1+\operatorname{erf}\left\{\frac{\ln \left(c / n_{c}\right)}{\sqrt{2} \sigma_{l}}\right\}\right]$

Exponential type: $P(c)=1-I \exp \left(-I \frac{c}{C}\right)$

where $\operatorname{erf}, n_{c}$ and $\sigma_{l}$ are error function, the median concentration and the logarithmic standard deviation. I stands for intermittency factor defined as a fraction of time, when no concentration signal appears and can be expressed using $C_{i}$ as follows. $^{16)}$

$$
I=\frac{2}{C_{i}^{2}+1}
$$

where $C_{i}$ is concentration fluctuation intensity defined as the ratio of r.m.s. concentration to mean concentration, $C_{r . m . s .} / C_{\text {ave }}$.

These theoretical models cannot predict the spatial distribution of concentration but can estimate peak concentrations at a stationary point. Sato et ll $^{17)}$ examined the lognormal and exponential types of probability distributions of concentration fluctuation behind an isolated cubical building with those for the wind tunnel experiment by using concentration statistics of $n_{c}, \sigma_{l}$ and $C_{i}$ obtained by the experiment. They showed that the probability distribution of concentration fluctuation can be evaluated by the lognormal type for $0.3<$ $C_{i}<1.0$, the lognormal or the exponential types for $1.0<C_{i}$ $<1.5$ and the exponential type for $C_{i}>1.5$. In this study, we compare to the wind tunnel experimental data of the probability distributions depending on the values of $C_{i}$ in order to examine our LES model performance.

Figure 9 shows time series of concentration at the central street canyon and crossing positions (see Figure 4). The red line indicates the averaged value. At the position of central street canyon, concentrations fluctuate smoothly and continuously. On the other hand, at the crossing position, instantaneous high concentrations frequently occur. These patterns of concentration fluctuation inside and outside of the cavity region of a building are similar to the wind tunnel experiment of Mavroidis and Griffiths. ${ }^{18)}$

Figure 10 shows a comparison of probability distributions $(1-P(c))$ of concentration fluctuation of LES at the central street canyon and crossing positions at the height of $\mathrm{z} / \mathrm{H}=0.1$. Concentration is normalized by $\mathrm{C}_{\mathrm{ave}} \cdot \mathrm{c}_{99}, \mathrm{c}_{95}$ and $\mathrm{c}_{90}$ are defined as the values determined from $1-P(c)=0.99$, 0.95 and 0.90 , respectively. Concentration fluctuation intensity, $C_{i}$, show 0.34 and 1.1 at the central street canyon and crossing positions, respectively. For evaluating probability distributions of concentration fluctuation of each theoretical model, concentration statistics of $n_{c}, \sigma_{l}$ and $C_{i}$ obtained by LES are used. At the position of central street canyon, the probability distribution of LES is consistent with that of the lognormal type, while that of LES is much smaller than the exponential one in the range of $0.01<1-P(c)<0.1$. At the crossing position, the probability distribution of LES is consistent with both lognormal and exponential types. It is shown from these LES results that the characteristics of probability distributions obtained by LES depending on the values of $C_{i}$ are consistent with those reported by Sato et $a l .{ }^{17)}$

Focusing on various peak concentrations, $\mathrm{c}_{99}, \mathrm{c}_{95}$ and $\mathrm{c}_{90}$ of LES at the central street canyon are found to show 2.0, 1.8 and 1.5 , respectively. On the other hand, those of LES at crossing position show 4.5, 3.8 and 2.7 , respectively, and qualitatively much different from those at the central street canyon. This indicates that it is important to evaluate peak concentrations based on a building scale for the assessment of human health hazard or the safety analysis of the hazardous gas within populated urban areas.

\section{Conclusion}

In this study, we perform Large-Eddy Simulation of plume dispersion within a regular array of cubic buildings as idealized urban canopy and investigate the characteristics of 

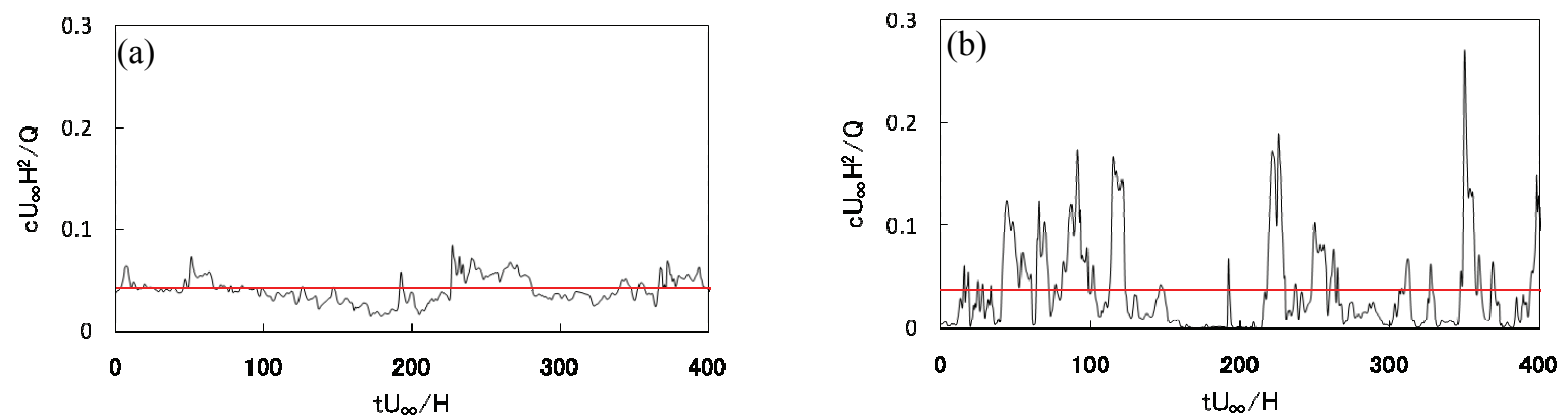

Fig. 9 Time series of concentration: (a) the central street canyon position and (b) the crossing position
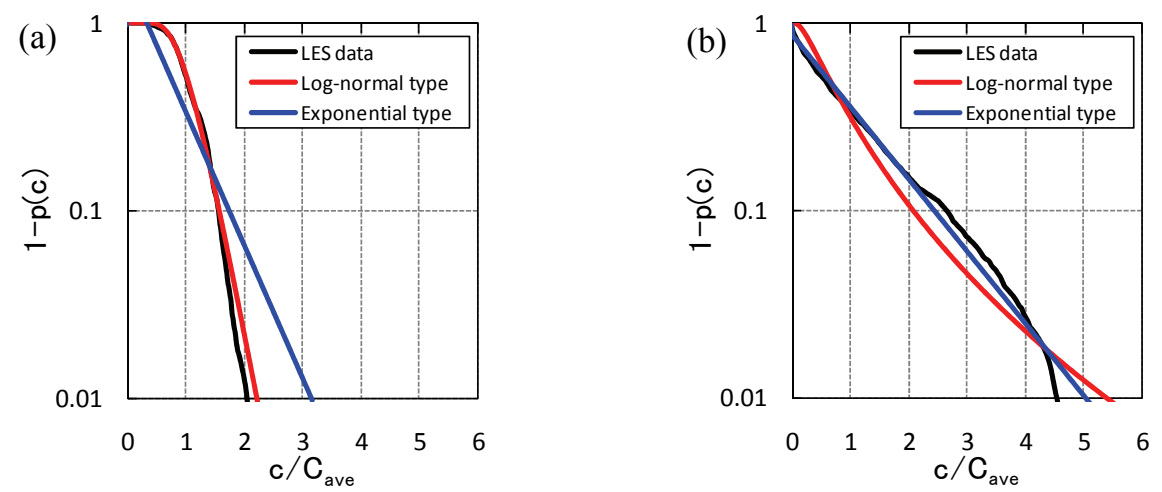

Fig. 10 Probability distribution functions of concentration: (a) the central street canyon position and (b) the crossing position

mean and fluctuating concentrations. The obtained results are as follows:

1. A spatially-developing turbulent boundary layer flow in the driver region is generated by incorporating existing inflow turbulence generation method into an upstream small fraction of the driver region with a tripping fence placed at the ground surface and roughness blocks. By this scheme, we have successfully simulated a turbulent boundary layer flow corresponding to the wind tunnel experiment of Bezpalcova and $\mathrm{Ohba}^{3)}$ in the driver section.

2. When compared to the experimental data ${ }^{3)}$ of the spanwise and vertical profiles of mean and r.m.s. concentrations, it is shown that this LES model successfully simulate plume dispersion within a building array. Furthermore, it is found that r.m.s. concentration rapidly decreases in the streamwise direction, while mean concentration gradually decreases with downwind distance.

3. It is shown that peak concentration values at the central street canyon are much different from those at crossing point. This indicates that it is important to evaluate peak concentrations based on a building scale for the assessment of human health hazard or the safety analysis of the hazardous gas within populated urban areas.

\section{Acknowledgment}

The authors thank Dr. Klara Bezpalcova who provided the wind tunnel experimental data.

\section{References}

1) R. Macdonald, R. Griffiths, S. Cheah, "Field experiments of dispersion through regular arrays of cubic structures," Atmos. Environ., 31[6], 783-795 (1997).

2) A. Sato, H. Takimoto, T. Michioka, M. Kanda, "Field Experiments of Flow and Dispersion within a Building Canyon of the Comprehensive Outdoor Scale Model," Ann. J. Hydraul. Eng., 53, 247-252 (2007), [in Japanese].

3) K. Bezpalcova, M.Ohba, "Advective and turbulent vertical fluxes of the passive contaminant inside an urban canopy," Proc. of 20th National Symposium on Wind Engi., Tokyo, Japan, 20, 19-24 (2008).

4) S. Oikawa, Y. Meng, "A Wind-tunnel Study of the Flow and Diffusion within Model Urban Canopies," J. Jpn. Soc. Atmos. Environ., 32, 148-156 (1997).

5) E. R. Van Driest, "On turbulent flow near a wall," J. Aero. Sci., 23, 1007-1011 (1956).

6) D. Goldstein, R. Handler, L. Sirovich, "Modeling a no-slip flow boundary with an external force field," J. Comput. Phys., 105, 354-366 (1993).

7) J. Smagorinsky, "General circulation experiments with the primitive equations,” Mon. Wea. Rev., 91[3] 99-164 (1963).

8) T. Shirasawa, Y. Endo, R. Yoshie, A. Mochida, H. Tanaka, "Comparison of LES and Durbin type k- $\varepsilon$ model for gas diffusion in weak wind region behind a building," J. Environ. Eng., 73[627], 615-622 (2008), [in Japanese]. 
9) K. Sada, A. Sato, "Numerical calculation of flow and stack-gas concentration fluctuation around a cubical building," Atmos. Environ., 36, 5527-5534 (2002).

10) A. J. Chorin, "A numerical method for solving incompressible viscous flow problems," J. Comp. Phys., 2, 12-26 (1967).

11) H. Takewaki, A. Nishiguchi, T. Yabe, "Cubic Interpolated Pseudo-particle method (CIP) for solving hyperbolic-type equations," J. Comput. Phys., 61, 261-268 (1985).

12) T. Yabe, E. Takei, "A new higher-order Godunov method for general hyperbolic equations," J. Phys. Soc, Japan, 57[8], 2598-2601 (1988).

13) H. Kataoka, M. Mizuno, "Numerical flow computation around aeroelastic 3D square cylinder using inflow turbulence," Wind Struc., 5, 379-392 (2002).
14) P. M, Gresho, "Some interesting issues in incompressible fluid dynamics, both in the continuum and in numerical simulation," Adv. Appl. Mech., 28, 45-140 (1992).

15) G. T. Csanady, "Turbulent diffusion in the environment," D. Reidel Publ., Dordrecht, Holland, 222-248 (1973).

16) S. R. Hanna, "The exponential probability density function and concentration fluctuation in smoke plumes," Bound-Layer Meteorol., 29, 361-375 (1984).

17) A. Sato, K. Sada, "A wind tunnel experiment on tracer gas concentration fluctuation near a cubical model building," Proc. Japan Soc. Civil Eng., 706, 41-49 (2002), [in Japanese].

18) I. Mavroidis, R. Griffiths, "Local characteristics of atmospheric dispersion within building arrays," Atmos. Environ., 35, 2941-2954 (2001). 